Rev Inv Vet Perú 2015; 26(4): 558-564

http://dx.doi.org/10.15381/rivep.v26i4.11209

\title{
Cuantificación de Micotoxinas en Ingredientes Alimenticios Utilizados en la Dieta de Aves Comerciales
}

\author{
Quantification of Micotoxins in Feedstuffs Used in Commercial Poultry Diets \\ Johnatan Castro ${ }^{1,2}$, Arnaldo Alvarado ${ }^{2,3}$, Ysabel Koga ${ }^{2,4}$, Robert Tinoco ${ }^{2}$
}

\section{Resumen}

\begin{abstract}
El estudio tuvo como objetivo cuantificar la presencia de las micotoxinas ocratoxina A y toxina T-2 en dos ingredientes alimenticios comunes en las dietas avícolas de procedencia diversa. Se analizaron 139 muestras de maíz y 64 de torta de soya para el contenido de ocratoxina A y 193 muestras de maíz y 144 de torta de soya para el contenido de toxina T-2, las mismas que fueron recibidas para análisis entre 2007 y 2011. Los resultados indicaron 66.2 y $67.4 \%$ de muestras positivas de maíz para ocratoxina A y toxina T-2, respectivamente. Asimismo, 71.9 y $88.9 \%$ muestras positivas de torta de soya para ocratoxina A y toxina T-2, respectivamente. Ninguna muestra llegó a sobrepasar los límites de ocratoxina A y T-2 permitidos, según las recomendaciones de la Comisión Europea (2006/576/EC).
\end{abstract}

Palabras clave: ocratoxina A, toxina T-2, maíz, harina de soya

\section{Abstract}

The study aimed to quantify the presence of micotoxins ochratoxine A and toxin T-2 in two common feedstuffs used in poultry diets (yellow corn and soybean meal) from different origins. Samples received for analysis from 2007 to 2011 were included in the study: 139 samples of corn and 64 of soybean meal were analyzed for ochratoxine A and 193 samples of corn and 144 of soybean were analized for toxin T-2. Results showed 66.2 and $67.4 \%$ positive samples of maize to ochratoxine A and toxin T-2 respectively, and 71.9 y $88.9 \%$ positive samples of soybean for ochratoxine A and toxin T-2 respectively. None of the samples surpassed the accepted limits recommended by the European Commission (2006/576/EC).

Key words: ochratoxine A, toxin T-2, corn, soybean meal

\footnotetext{
${ }^{1}$ Facultad de Medicina Veterinaria, Universidad Nacional San Luis Gonzaga de Ica, Perú

${ }^{2}$ Bioservice SRL, Lima, Perú

${ }^{3}$ Laboratorio de Patología Clínica y Biología Molecular, Facultad de Medicina Veterinaria, Universidad Nacional Mayor de San Marcos, Lima, Perú

${ }^{4}$ E-mail: ykogay@hotmail.com
}

Recibido: 5 de febrero de 2015

Aceptado para publicación: 19 de mayo de 2015 


\section{INTRODUCCIÓN}

La FAO (2004) define a las micotoxinas como metabolitos de hongos que provocan cambios patológicos, tanto en seres humanos como animales, y a la micotoxicosis como los síndromes de la toxicidad resultante de la absorción de micotoxinas. El término micotoxina deriva de las palabras griegas «mykes» (hongos) y «toksicons» (veneno) (Torres et al., 1995).

Las micotoxinas son producidas en la etapa final del crecimiento exponencial de una colonia fúngica y sin importancia aparente para el crecimiento o metabolismo de estos organismos (Torres et al., 1995). Diferente es el caso de los metabolitos primarios que son esenciales para el crecimiento del microorganismo (Jay, 2000). Las micotoxinas son un grupo muy amplio de metabolitos tóxicos para los animales y para el hombre; toxicidad que puede variar desde el desarrollo de actividades carcinógenas, teratógenas o mutágenas hasta la producción de desórdenes de tipo hormonal o inmunosupresor $y$, dependiendo de la micotoxina, puede incluso ocasionar la muerte (De Lange et al., 2000).

La contaminación de granos por micotoxinas es común. El Council for Agricultural Science and Technology CAST (2000) señala que cerca de $25 \%$ de los alimentos cosechados anualmente a nivel mundial se encuentran afectados por micotoxinas; en tanto que Sokoloviæ et al. (2008) indica que el nivel de contaminación varía entre el 25 y el $40 \%$.

Las micotoxinas son ingeridas con los alimentos o forrajes contaminados directa o indirectamente. La contaminación directa con un moho, y la consecuente producción de toxina, puede ocurrir durante la producción, transporte, estacionamiento o procesamiento del alimento o forraje, mientras que la contaminación indirecta se debe a la presencia de un ingrediente previamente contaminado con un moho toxinogénico que ya ha desaparecido pero cuya micotoxina persiste (Diekman y Green, 1992).

Se han identificado más de 200 tipos de micotoxinas. Sin embargo, las que pueden encontrarse con mayor frecuencia como contaminantes naturales en los alimentos para animales o humanos son las aflatoxinas B1, B2, G1, G2 y M1, ocratoxinas, zearalenona, tricotecenos (vomitoxina, T-2, nivalenol, DON), citrinina, patulina y fumonisinas B1 y B2 (Ready y Ready, 1992).

Las ocratoxinas son un grupo de metabolitos tóxicos, producidas por varias especies de los géneros Aspergillus y Penicillum. La ocratoxina A, que es la más tóxica, es una nefrotoxina que causa hipertrofia y decoloración del riñón y acumulación de ácido úrico en humanos y cerdos. En aves se observa reducción en el crecimiento, disminución de la pigmentación y alteración en los procesos de coagulación sanguínea, y problemas de patas en el caso de pollos de engorde. Causa, además, inmunosupresión. A nivel celular, la ocratoxina A inhibe la síntesis de proteína (Etzel, 2002). Durante la intoxicación aguda, los animales están deprimidos, con anorexia, ascitis, y con edemas subcutáneos y mesentéricos. En la intoxicación crónica disminuye el apetito y el crecimiento, aumenta el consumo de agua y aparece poliuria.

Se conoce más de 100 tricotecenos, donde casi la mitad son producidos por hongos del género Fusarium. La mayor producción de la toxina se desarrolla con humedad elevada y temperaturas entre 6 y $24^{\circ} \mathrm{C}$. La toxina T-2, el diacetoxiscerpenol (DAS), el deoxinivalenol (DON), vomitoxina y el nivalenol han sido identificadas en el maíz, trigo, cebada, avena, arroz, centeno, sorgo, azafrán, alimentos mezclados y granos cerveceros (Bock et al., 1986). Han causado brotes de enfermedad hemorrágica en animales y están relacionados a lesiones bucales y efectos neurotóxicos en aves de corral, siendo el efecto más importante su actividad inmunodepresora (Dänicke et al., 2007). 
Intoxicaciones crónicas en aves que involucran la toxina T-2 o diacetoxiscerpenol (DAS) inducen a una reducción del consumo de alimento balanceado y ganancia de peso, lesiones orales, necrosis en el tejido, hematopoyético y mucosa oral, con eventuales trastornos nerviosos (posición anormal de las alas, reducción de reflejos), emplume anormal y reducción del espesor de la cáscara de los huevos. En ponedoras, las lesiones orales se producen en aproximadamente el $50 \%$ de los lotes o parvadas cuando se les proporciona alimento balanceado que contiene 2 ppm de toxina T-2. Esta toxina también induce la formación de peróxidos a partir de los lípidos, acarreando la disminución de la concentración de vitamina $\mathrm{E}$ en las aves (Mallmann et al., 2007).

Otras aves, como pavos y gansos, son más sensibles a la toxina T-2 que los pollos de engorde (Mallmann et al., 2007). También es posible observar una reducción del consumo de alimento balanceado en contaminaciones por toxina T-2 (Brake et al., 2002).

Existen varios métodos analíticos para la determinación de micotoxinas, entre ellos la técnica de ELISA competitiva, cuyo principio básico es la afinidad de la micotoxina por anticuerpos específicos fijados a un soporte, algunas de las cuales permiten el control de las micotoxinas in situ de un modo rápido, fiable y sencillo (Shell et al., 1993).

El objetivo del presente estudio fue identificar y cuantificar las micotoxinas presentes en el alimento balanceado de aves que se comercializa en el Perú.

\section{Materiales y Métodos}

El presente estudio se llevó a cabo en el área de Toxicología del Laboratorio Bioservice S.R.L., ubicado en Villa María del Triunfo, Lima, Perú, entre los meses de enero y agosto de 2012.
La investigación fue considerada de tipo descriptiva, longitudinal y retrospectiva. Se utilizó la información resultante de muestras enviadas al laboratorio por diversas certificadoras durante los años 2007 a 2011. El alimento, cuyas muestras fueron enviadas al laboratorio, estaba destinado al sector pecuario, básicamente al sector avícola. Las muestras procedían de países tales como Paraguay, Argentina, Brasil y Estados Unidos.

Cada muestra consistía entre 500 y 1000 $\mathrm{g}$, tanto para análisis de rutina como específicamente para determinación de la toxina T-2 u ocratoxina. Así, 139 muestras de maíz fueron analizadas para el contenido de ocratoxina A y 193 para el contenido de toxina T-2. Asimismo, 64 muestras de torta de soya fueron analizadas para el contenido de ocratoxina A y 144 muestras para toxina $\mathrm{T}-2$.

Las muestras fueron sometidas a un ensayo de ELISA con kits comerciales (Ridascreen ${ }^{\circledR}$ Fast Ochratoxin A y T-2 toxin, ambos de R-BiopharmAG, Alemania). El procedimiento y análisis de los resultados fueron realizados según las recomendaciones del fabricante. No obstante, la muestra se consideró positiva a micotoxina con la sola presencia de ella y no necesariamente que el valor haya pasado el límite recomendable.

Los datos obtenidos fueron tabulados, identificando y cuantificando las micotoxinas por tipo y por ingrediente alimenticio para aves comerciales.

\section{Resultados}

En el Cuadro 1 se observa que el 66.2 y $71.9 \%$ de las muestras de maíz y torta de soya, respectivamente, tenían algún nivel de contaminación con ocratoxina. Asimismo, el 67.4 y $88.9 \%$ de las muestras de maíz y torta de soya, respectivamente, tenían algún nivel de contaminación con toxina T2. 
Cuadro 1. Niveles de ocratoxina y toxina T-2 en maíz y torta de soya destinado para alimentación de aves de corral (2007-2012)

\begin{tabular}{llccccc}
\hline \multirow{2}{*}{ Ingrediente } & Micotoxina & \multicolumn{3}{c}{ Muestras } & \multicolumn{3}{c}{ Valores (ppb) } \\
\cline { 3 - 7 } & & $\begin{array}{c}\text { Total } \\
(\mathrm{n})\end{array}$ & $\begin{array}{c}\text { Positivas } \\
(\%)\end{array}$ & $\begin{array}{c}\text { Promedio } \pm \\
\text { d.e. }\end{array}$ & Mínimo & Máximo \\
\hline \multirow{2}{*}{ Maíz } & Ocratoxina & 139 & 66.2 & $1.83 \pm 1.66$ & 0.10 & 7.78 \\
& Toxina T2 & 193 & 67.4 & $10.78 \pm 11.29$ & 0.07 & 79.41 \\
\multirow{2}{*}{ Torta de soya } & Ocratoxina & 64 & 71.9 & $1.99 \pm 1.63$ & 0.02 & 5.99 \\
& Toxina T2 & 144 & 88.9 & $12.96 \pm 9.97$ & 0.08 & 47.33 \\
\hline
\end{tabular}

\section{Discusión}

Varios organismos internacionales están tratando de lograr una estandarización universal de los límites reglamentarios para las micotoxinas. Esta es una tarea difícil puesto que deben considerarse muchos factores para decidir las normas reglamentarias. Además de los factores científicos tales como la evaluación del riesgo y la exactitud analítica, hay factores económicos y políticos, tales como los intereses comerciales de cada país y la permanente necesidad de un suministro suficiente de alimentos, que también juegan un papel en el proceso de toma de decisiones.

El proceso se complica aún más por el hecho de que las micotoxinas suelen presentarse en combinación y de esta forma los niveles de acción son más bajos. Medir los efectos toxicológicos de una diversidad de combinaciones de micotoxinas, tal como se presentan en la naturaleza, es una tarea complicada, especialmente si se considera que puede haber micotoxinas aún no identificadas. Además, la nutrición, la gestión, el ambiente y las especies contribuyen a determinar el efecto de la combinación de micotoxinas sobre la salud animal (KnowMycotoxins, 2012).

A pesar de estos desafíos, los países de la Unión Europea han llegado a un acuerdo común sobre una política estandarizada para la regulación de los principales niveles de micotoxinas en distintos alimentos e ingredientes de alimentos. Los niveles de tolerancia en esta sección se basan fundamentalmente en estudios sobre toxinas individuales. Investigaciones adicionales sobre las interacciones de las micotoxinas entre sí y con otros factores ambientales y nutricionales permitirán la validación y modificación de estas pautas (KnowMycotoxins, 2012).

La Comisión Europea (2006/576/EC) ha dado algunas recomendaciones sobre la presencia de micotoxinas, dentro de las que menciona ocratoxina A y toxina T-2 en productos destinados a alimento animal. Esta comisión considera algunos puntos claves como incrementar el monitoreo para identificar la presencia de Deoxinivalenol (DON), Zearalenona (ZEA), Ocratoxina (OTA), Fumonisina (FUM) B1 y B2, Toxina T2 (T2) y Toxina HT-2 en cereales y productos diseñados para la alimentación animal y alimentos compuestos para permitir la evaluación del alcance de la coexistencia de múltiples micotoxinas.

En este contexto, la Comisión Europea considera valores máximos de ocratoxina $\mathrm{A}$ de $0.1 \mathrm{ppm}$ para alimentos complementarios y completos para aves y de 0.05 ppm para alimentos complementarios y completos para cerdos. Sin embargo, esta comisión reporta valores límites diferentes para el caso de otros 
países como Israel, Canadá, China, Uruguay y Ucrania para ocratoxina A y toxina T2, lo que comparado con los resultados del presente estudio, indica que los valores promedios encontrados están por debajo de las recomendaciones límites para ambas micotoxinas en maíz y torta de soya.

Otro factor importante a considerar es que usualmente la presencia de DON y toxina $\mathrm{T}-2$ es indicativa de que existen docenas de otras micotoxinas igualmente tóxicas como los tricotecenos del Fusarium (DeVries et al., 2002, citado por Haladi, 2012). De ser así, se podría asumir que, de acuerdo al elevado porcentaje de muestras positivas de las micotoxinas evaluadas, habría paralelamente otras micotoxinas no evaluadas en las muestras trabajadas. Zafar et al. (2001) encontraron el 23\% (36/156) de muestras de ingredientes alimenticios contaminados con ocratoxina, donde el contenido más alto en el maíz fue de $104.4 \mathrm{ppb}$, mientras que en la harina de soya fue de $31 \mathrm{ppb}$.

También se debe considerar las interacciones sinérgicas entre AFLA y OTA (Huff y Doer, 1981; Huff et al., 1983), entre OTA y T-2 (Huff et al., 1988) y una interacción antagónica entre OTA y DAS (Kubena et al., 1997) observadas en pollos de engorde.

Por otro lado, también es necesario considerar el método y equipo utilizado para el análisis de las micotoxinas. En este sentido, Rodrigues y Naehrer (2012), analizando diversos insumos alimenticios $(\mathrm{n}=1695)$ por el método de ELISA, encontraron el 24, 21, 65 , 48 y $17 \%$ de muestras contaminadas con aflatoxina, dioxinivalenol, zearalenona, fumonisina y ocratoxina A, respectivamente. Además, se encontró el $47 \%$ de muestras contaminadas (sin considerar límites permisibles) por al menos una micotoxina y el $18 \%$ con dos o más micotoxinas. Asimismo, en dicho estudio se evaluaron 12 muestras de maíz y 4 muestras de torta de soya por el método de ELISA, se encontró 8 y $25 \%$ de muestras positivas a ocratoxina A con un nivel promedio de 1 y 5 ppb y un nivel máximo de 11 y
21.4 ppb para las muestras positivas de maíz y torta de soya, respectivamente.

En un estudio hecho en Brasil por Mallmann et al. (2007) se encuentra el 2.9\% de positividad con un nivel promedio de 0.6 ppb de ocratoxina A en 19730 muestras y de $1.3 \%$ de positividad con un nivel promedio de 13.9 ppb de toxina T2 en 10952 muestras. Estos investigadores, basados en estudios propios y en resultados de muestras en el Laboratorio de Análisis de Micotoxinas (LAMIC) de la Universidad Federal de Santa María, Brasil, recomendaron límites de seguridad de $0,2,5,5$ y 5 ppb para ocratoxina y de 0,50 , 50,100 y $100 \mathrm{ppb}$ para la toxina $\mathrm{T}-2$ en pollos de engorde en fase inicial, fase de crecimiento, fase final, ponedoras comerciales y reproductoras, respectivamente. Sin embargo, otros expertos no hacen diferencia entre las diferentes etapas productivas de las aves y consideran que el nivel máximo para ocratoxina es $5 \mathrm{ppb}$ y para toxina T-2 es de 100 ppb (Zaviezo, 2012).

Las muestras trabajadas en este estudio fueron remitidas por clientes del laboratorio para análisis de rutina, y se debe tener en cuenta que proceden de varios países y con diferentes formas de conservación y antigüedad. Si bien es cierto que los valores obtenidos no exceden los límites máximos permisibles, hay que tener en cuenta que el proceso de intoxicación con micotoxinas es de tipo crónico, de allí que aún con niveles bajos, su acumulación en el organismo del ave puede llegar a generar problemas productivos. Asimismo, se debe tomar en cuenta la coexistencia de otros tricotecenos que no se analizaron.

Si bien existen datos referenciales sobre los límites permisibles o máximos para micotoxinas, cada laboratorio debe tener su propia línea de base de datos y, de acuerdo a lo acontecido en el campo, determinar cuáles son sus valores permisibles. Muchas veces no se pueden comparar resultados entre laboratorios porque están implicados diversos factores individuales que hacen difícil su in- 
terpretación. En ese sentido y de acuerdo a la casuística de estudios realizada entre los años 2001 y 2006 en Bioservice SRL, valores mayores o iguales a $10 \mathrm{ppb}$ y $100 \mathrm{ppb}$ para ocratoxina A y T-2 produjeron daños a nivel renal y hepático en las aves, lo cual fue corroborado mediante pruebas bioquímicas e histopatológicas, así como por disminución de los parámetros productivos.

\section{Conclusiones}

- $\quad$ El 71.9 y $88.9 \%$ de las muestras de maíz $\mathrm{y}$ torta de soya resultaron positivas para ocratoxina A, y el $66.267 .4 \%$ de estos insumos resultaron positivos para la toxina $\mathrm{T}-2$, respectivamente.

- Los niveles promedios de ocratoxina y T-2 en maíz y soya estuvieron por debajo de los límites permitidos según las recomendaciones de la Comisión Europea (2006/576/EC), no habiendo muestras que superaron los límites permitidos

\section{Literatura Citada}

1. Bock RR, Shore LS, Samberg Y, Perl $S$. 1986. Death in broiler breeders due to salpingitis: possible role of zearalenone. Avian Pathol 15: 495-502. doi: 10.1080/03079458608436310

2. [CAST] Council for Agricultural Science and Technology. 2003. Mycotoxins: risk in plant, animal and human systems. Task Force Report. Iowa, USA: CAST. 199 p.

3. Dänicke S, Valenta $H$, Matthes $S$. 2007. On the interactions between Fusarium toxin-contaminated wheat and nonstarch polysaccharide hydrolyzing enzymes in diets of broilers on performance, intestinal viscosity, and carryover of deoxynivalenol. Poultry Sci 86: 291298. doi: 10.1080/00071660601148161

4. De Lange $C$, Nyachoti $C$, Verstegen M. 2000. The significance of antinutritional factors in feedstuffs for monogastric animals. En: Moughan P,
Verstegen M, Visser-Reyneveld M (eds). Feed evaluation principles and practice. Amstelveen, Holanda: Wageningen Pers. p 169-188.

5. Diekman H, Green K. 1992. Mycotoxins and reproduction in domestic livestock. J Anim Sci 70: 1615-1627.

6. Etzel R. 2002. Mycotoxins. J Am Med Assoc 287: 425-427. doi:10.1001/ jama.287.4.425

7. The Commission of the European Communities. 2006. The Commission Recommendation of 17 August 2006 on the presence of deoxynivalenol, zearalenone, ochratoxin A, T-2 and HT2 and fumonisins in products intended for animal feeding (2006/576/EC). Official Journal of the European Union. [Internet]. Disponible en: http://eurlex.europa.eu/LexUriServ/ LexUriServ.do?uri=OJ:L:2006: 229:0007:0009:EN:PDF

8. Haladi S. 2012. Micotoxicosis en aves. Boletin Alltech. 3 p.

9. Jay J. 2000. Modern food microbiology. $6^{\text {th }}$ ed. USA: Aspen Publication. 679 p.

10. Huff WE, Doerr JA. 1981. Synergism between aflatoxin and ochratoxin A in broiler chickens. Poultry Sci 60:550-551.

11. Huff WE, Doerr JA, Wabeck CJ, Chaloupka GW, May JD, Merkley $J W$. 1983. Individual and combined effects of aflatoxin and ochratoxin on bruising in broiler chickens. Poultry Sci 62: 1764-1771. doi: 10.3382/ps.0621764

12. Huff WE, Harvey RB, Kubena LF, Rottinghaus GE. 1988. Toxin synergism between aflatoxin and T-2 toxin in broiler chickens. Poultry Sci 67: 1418- 1423.

13. Know Mycotoxins. 2012. Reglamentaciones. [Internet]. Disponible en: http:// www.know mycotoxins.com/es/ regulations.htm

14. Kubena LF, Edrington TS, Harvey RB, Phillips TD, Sarr AB, Rottinghaus

$\boldsymbol{G E}$. 1997. Individual and combined effects of fumonisin B1 present in Fusarium moniliforme culture material and diacetoxyscirpenol or ochratoxin A in turkey poults. Poultry Sci 76: 256-264. 
15. Mallmann CA, Dilkin P, Giacomini LZ, Rauber RH, Pereira CE. 2007. Micotoxinas en ingredientes para alimento balanceado de aves. En: XX Congreso Latinoamericano de Avicultura. Porto Alegre, Brasil.

16. Ready GL, Ready SM. 1992. Production of roridin by Myrothecium raridum in mixed culture. Indian $\mathbf{J}$ Microbiol 31: 281-284.

17. Rodrigues I, Naehrer K. 2012. Prevalence of mycotoxins in feedstuffs and feed surveyed worldwide in 2009 and 2010. Phytopathologia Mediterranea 51: 175-192. doi: http://dx.doi.org/10.14601/ Phytopathol_Mediterr-9693

18. Schell T, Lindemann M, Kornegay EY, Blodgett D. 1993. Effects of feeding aflatoxin-contaminated diets with and without clay to weanling and growing pigs on performance, liver function, and mineral metabolism. J Anim Sci 71: 1209-1218.

19. Sokolovia M, Garaj-Vrhovac V, Simpraga B. 2008. T-2 toxin: incidence and toxicity in poultry. Arh Hig Rada Toksikol 59: 43-52. doi: 10.2478/100041254-59-2008-1843

20. Torres E, Acuna K, Naccha LR, Montoya R, Castrellon JP. 1995. Quantification of aflatoxins in corn distributed in the city of Monterrey, Mexico. Food Addit Contam 12: 383-386.

21. Zafar F, Yasmin N, Hassan R, Naim T, Qureshi AA. 2001. A study on the analysis of ochratoxin A in different poultry feed ingredients. Pak J Pharm Sci 14: 5-7.

22. Zaviezo D. 2012. Consideraciones técnicas sobre la problemática de micotoxinas y las micotoxicosis aviares. Cienc Trabajo 8(22): 154-158. 